Archivum, LXXI, 2021, pp. 475-503

\title{
Acercamiento a lenguas amerindias extintas a través de documentación archivística*
}

\author{
Bojana Radosavljevic \\ Universidad de Málaga \\ bojana.radosavljevi3@gmail.com
}

Recibido: 14/06/2021

Aceptado: 29/09/2021

\section{RESUMEN:}

El escrito que se desarrolla en estas páginas se propone analizar algunos vocablos de las lenguas extintas pericú, cochimí y guaycura, como son aynu, boxo, riyero o medesá, que fueron documentados en los textos castellanos de data del siglo XVII y autoría del almirante de Las Californias don Isidro de Atondo y Antillón. Los textos tomados para realizar este escrito fueron hallados en tres recopilaciones tituladas Descubrimiento en Las Californias, Autos sobre la última entrada en Las Californias y Cartas del virrey marqués de la Laguna que se conservan en el Archivo General de Indias. El estudio de estos vocablos se complementa con el análisis de otras palabras, no menos importantes, con las cuales se designan las naciones observadas en sus textos, todas ellas bajacalifornianas y de cuyas lenguas proceden los ejemplos destacados. De ese modo se pone de manifiesto la escasa documentación que existe de dichas lenguas y palabras, tanto en los testimonios de navegantes o misioneros como en los estudios académicos; y con ello

* Todas las transcripciones que se hallan en este artículo fueron realizadas por la autora. 
se da paso al cumplimiento de otro objetivo de este análisis, consistente en transcribir los fragmentos que incluyen estas voces para dar a conocer los periplos del almirante, la cultura de dichas naciones, la toponimia perdida en las lenguas indígenas y la conciencia de los conquistadores de estar dándole nombre a algo que ya lo tiene.

PALABRAS CLAVE: lenguas extintas, Isidro de Atondo y Antillón, Baja California, edición crítica.

\title{
Approach to extinct Amerindian languages through archival documentation
}

\begin{abstract}
:
The writing that takes place in these pages aims to analyze some words from the extinct languages Pericú, Cochimí and Waikura, such as aynu, boxo, riyero or medesá, which were documented in Spanish texts dating from the 17th century and authorship of the Admiral of the Californias don Isidro de Atondo and Antillón. The texts taken to carry out this writing were found in three compilations entitled Descubrimiento en Las Californias, Autos sobre la última entrada en Las Californias and Cartas del virrey marqués de la Laguna that are preserved in the General Archive of the Indies. The study of these words is complemented by the analysis of other words, no less important, with which the nations observed in his texts are designated, all of them native Baja Californians and from whose languages the outstanding examples come. In this way, the scarce documentation that exists of these languages and words is revealed, both in the testimonies of navigators or missionaries and in academic studies; and with this, another objective of this analysis is fulfilled, consisting of transcribing the fragments that include these voices to make known the admiral's journeys, the culture of these nations, the lost toponymy in indigenous languages and the awareness of the conquerors of giving a name to something that already has it.
\end{abstract}

KEYWORDS: extinct languages, Isidro de Atondo y Antillón, Baja California, critical edition.

\section{Introducción}

Desde el siglo XVIII hasta la actualidad el Archivo General de Indias (AGI) ha mantenido su interés por reunir, catalogar rigurosamente y preservar los documentos que testimonian la historia de los territorios desde España y el Nuevo Mundo hasta Estados 
Unidos y Filipinas. La magnífica colección de obras manuscritas e incunables reunidas por el AGI que abarcan esta inmensa extensión geográfica y una época que comprende más de tres siglos se preserva dispuesta en estanterías de más de ocho kilómetros que contienen 80 millones de folios de textos originales, en los que se halla un amplio abanico de temas relacionados como las evangelizaciones, las administraciones llevadas a cabo en estos territorios para la época, el tráfico marítimo o la antropología, por señalar algunos (Lázaro de la Escosura y Ceballos Aragón, 2008, 9 y ss.). Esta colección ha deparado el hallazgo del grupo de manuscritos de data del siglo XVII que son objeto de estudio en este artículo.

Hay documentos en el Archivo que pasan por la sala de lectura a lo largo del año más de cien veces, lo que obviamente plantea unos gravísimos problemas para su buena conservación [...]. Es claro que hay que mantener e incrementar el acceso a la información, pero también que hay que salvaguardar el patrimonio histórico que nos ha sido legado [...]. (González, 1994, 242)

Atendiendo este deber de salvaguardar el patrimonio, con la motivación de mantener su buena conservación, el AGI puso en marcha desde hace algunos años un proyecto de digitalización de su colección de obras, tarea que, además de su preservación, permite ampliar el acceso a los documentos archivados que son interés de investigadores. Esta colección fue consultada mediante una búsqueda digital para elaborar el presente artículo, resultado de la cual se obtuvieron una serie de cartas, diarios, testimonios, autos, certificaciones y peticiones fechadas en el siglo XVII y hechos por el almirante de Las Californias don Isidro de Atondo y Antillón.

\section{Biografía del almirante de Las Californias don Isidro de Atondo y Antillón}

Isidro de Atondo y Antillón (Hernández Aparicio, 1980, 3-43; Mathes, 1969, 211-218; Rodríguez Tomp y Almada, 2014, 92-100; 
Ibarra Rivera, 2011, 58-110) nació en la villa de Valtierra (Navarra) y fue bautizado en el año 1639. Fue nombrado almirante de Las Californias y gobernador de Sinaloa, además, prestó su servicio como soldado en el Ejército de Galicia con Pedro Nuño Colón a bordo de las naves Isabel y San Salvador en la guerra de independencia de Portugal. El título de almirante le fue otorgado a Isidro de Atondo y Antillón en el año 1678, por el virrey fray Payo Enríquez de Rivera.

Después de fallidas expediciones realizadas por Bernal Bernardo Piñadero y tras haber trascurrido un año de recibir su nombramiento, en Guasave (México), Isidro de Atondo y Antillón empezó con las preparaciones para emprender su misión de conquistar y poblar la Baja California. Su tarea de conquista la llevó a cabo junto con el padre Eusebio Francisco Kino de la Compañía de Jesús, quien fue un héroe de México y Arizona, considerado también un brillante misionero y científico de la época. Las misiones de conquista de Isidro de Atondo y Antillón y Eusebio Francisco Kino tuvieron lugar en el puerto de La Paz, al que atracaron en el año 1683 las embarcaciones La Concepción, San José y San Francisco Javier. Desde ese entonces y hasta el fin de las expediciones en el año 1685 se fundaron los reales de San Bruno, San Dionisio y San Isidro; además, se descubrieron el puerto de Danzantes y las islas del Carmen, de San José y de los Santos Coronados, entre otros lugares que al ser descubiertos recibieron distintas denominaciones que se han anotado en los respectivos paréntesis que los acompañan como fueron: Adluiruitto (San Simón), Bunmedojol (San Gabriel), Nebocojol (Santa María), Tuirú (San Sereno), Damadamuyete (La Concepción) y muchos otros.

La biografía de Isidro de Atondo y Antillón muestra, en síntesis, que el almirante fue un hombre de brillante vida militar en la Península Ibérica y la Baja California (México), con muchas calidades, entre las que destaca el rescate del Galeón de Manila del posible ataque de piratas holandeses en su camino a Acapulco. Es por eso por lo que al terminar su vida militar 
recibió el nombramiento de caballero de la Orden de Santiago en el año 1689.

\section{Características del corpus de don Isidro de Atondo y Antillón}

El corpus de los textos elaborados por don Isidro de Atondo y Antillón está constituido por los manuscritos originales del almirante, los cuales se hallan en las recopilaciones que datan de finales del siglo XVII que son custodiadas en el AGI y llevan por títulos Descubrimiento en Las Californias (marzo de 1685), Autos sobre la última entrada en Las Californias (agosto de 1685) y Cartas del virrey marqués de la Laguna (marzo de 1686). Las primeras dos recopilaciones se conservan en la sección de Patronato del Archivo y la tercera es preservada en la sección de México.

Los documentos del almirante que han sido consultados se encuentran localizados en el caso del Descubrimiento en Las Californias en los folios 836v, 840v-847r, 847r-848v, 857r, 861r-861v, 862r-863v, 865v-867r, 867r-870r, 873r, 873r-876r, 877v, 877v-878v, $883 \mathrm{r}-885 \mathrm{r}, 885 \mathrm{r}-886 \mathrm{v}, 886 \mathrm{v}-889 \mathrm{v}, 889 \mathrm{v}-890 \mathrm{v}, 890 \mathrm{v}-893 \mathrm{r}, 893 \mathrm{r}-894 \mathrm{v}$ y 895r-897r. En los Autos sobre la última entrada en Las Californias los documentos se albergan en los folios 956r-956v, 960r-960v, 960v, 961r-994r, 994r-995r, 995r-1035r, 1035r-1035v, 1066v-1072v, 1072v y 1075v-1078r. Y en el caso de las Cartas del virrey marqués de la Laguna, donde los archivos se enumeran según números simples y en ocasiones en rectos y versos, los documentos están disponibles en los folios 1-7, 7-14, 14-76, 76-78, 78-79, 79-80, 127152, 153r-156v, 157 y $157-159 .{ }^{1}$

Es importante decir que los documentos del almirante que hacen parte de la colección del Archivo se encuentran en buen estado de conservación y sin tachaduras, correcciones o añadi-

1 Para la elaboración de este artículo se estudiaron estos folios en función de la localización de las palabras que se analizan: en el Descubrimiento en Las Californias, ff. 846v (medese), 862r (didiu), 863r-863v (didiu, edú), 887r (didiu), 888r-888v (medese) y 890r (medese); en los Autos sobre la última entrada en Las Californias, ff. 962v (edú), 964v (edú), 971v (edú), 975r (moquis), 982v (moquis, edúes), 985r (moquis), 999v (didiu) y 1067r (edú, didiu); y en las Cartas del virrey marqués de la Laguna, ff. 34 (aynu), 36 (boxoo), 44 (edú), 56 (riyero), 127 (didiu), 128 (edú, guicura), 130 (didiu) y 144 (didiu, edú). 
dos a lo largo de sus folios. Su soporte es el papel, el cual fue considerado un soporte jurídico y documental de gran valor durante muchos siglos por la Corona española (Rojas García, 2016), tal y como lo ejemplifica este corpus, puesto que era muy útil para conocer y cumplir órdenes y transmitir información sobre los resultados de la conquista y la población de la Baja California en los años ochenta del siglo XVII. Finalmente, cabe mencionar que todos los textos están escritos en castellano, en letra humanística.

\section{Corpus del almirante: lenguas extintas}

Desde hace algunas décadas, especialmente hoy en día, se ha ido recogiendo una abundante documentación académica referida a la historia y la arqueología de la Baja California, tarea en la que sobresale William Michael Mathes (Busto-Ibarra, 2015, 91-92). Sin embargo, en el plano lingüístico se advierte una limitación en la disponibilidad de datos sobre las lenguas indígenas de la Baja California, en especial sobre el idioma pericú (León-Portilla, 1976, 87).

Las lenguas que se presentan en este artículo son: la lengua cochimí, hablada por la nación cochimíes (a veces llamados didios; vid. 6.4), en el territorio que comprende desde el extremo norte de la península hasta la desembocadura del río Colorado; la lengua pericú, hablada por la nación que vivía en el extremo sur de la Baja California y se extendía hacia el Cabo San Lucas y Cabo Pulmo en el norte, conocida como edúes y nombrada pericúes o pericos por los españoles por su forma de hablar (Holmos Montaño, 2018, 25); y la lengua guaycura ${ }^{2}$, en la media, hablada por la nación guaycura que se ubicaba al norte del territorio pericú hasta la ciudad de Loreto (quienes hacían parte de la nación guaycura del Puerto de Danzantes eran llamados edúes por los cochimíes, de ahí la importancia de no confundir con los edúes

2 La nación guaycura, también conocida como vaicuras y monquis (guaycuras de Loreto o loretanos), estaba dividida en los subgrupos: callejúes (los guaycuras de La Paz), huchitíes, aripes, pecunes y vines (Holmos Montaño, 2018, 26). 
como pericúes $\left.{ }^{3}\right)$. Estas lenguas se encuentran en proceso de extinción desde el siglo XVIII, o bien han desaparecido como lo señaló Michael Wilken $(2009,5)$ en su artículo con estas palabras

After thousands of years, the languages that once could be heard throughout the deserts, coasts, and hillsides of the peninsula have all but disappeared, and those that remain hang by a thread. The Cochimi, Guaicura, and Pericú languages (...) are long gone, victims of the early onslaught of contagious diseases and cultural displacement that came with the Spanish conquest of the territory. The few remaining native speakers are mostly elderly members of the surviving Cucapá, Kiliwa, Paipai ${ }^{5}$, and Kumiai communities in the northern regions of the peninsula (...).

La desaparición de estas lenguas es bien conocida desde hace tiempo atrás como lo atestigua el testimonio de Francisco Javier Clavijero (1980 [1852], 22), jesuita del siglo XVIII, en el caso del idioma pericú, quien explicó que "la lengua pericú ya no existe, y los pocos individuos que han quedado de aquella desgraciada nacion hablan hoy la española". De hecho, entre las primeras documentaciones que se realizaron de las lenguas indígenas de la península de la Baja California se encuentran los testimonios de los misioneros ${ }^{6}$, entre los que destaca Francisco Javier Clavijero (ídem) que documentó las lenguas cochimí, guaycura y pericú, así como también las naciones que las hablaban:

3 En adelante, en el artículo se indicará donde proceda la respectiva denominación "edúes (pericúes)" o "edúes (guaycuras)", con el objetivo de evitar confusiones entre estas.

4 Apreciación también recogida en León-Portilla (1976, 87; 2000, 67-68), Holmos Montaño $(2018,21)$, Laylander (1985), y en Golla $(2011,59)$ en el caso de cochimí y guaycura.

5 El mapa de Survey of California and Other Indian Languages de la Universidad de California, Berkeley, registró las lenguas paipai (Akwa'ala) y kiliwa, ambas habladas en el norte de la península.

6 En cuanto a las otras documentaciones secundarias, estas la constituyen los documentos y textos escritos por navegantes del siglo XVII, como es el caso de Isidro de Atondo y Antillón. 
Los pericúes ocupan la parte austral de la península desde el Cabo de San Lúcas hasta los $24 .^{\circ}$ y las islas adyacentes de Cerralvo, el Espíritu Santo y San José; los guaicuras se establecieron entre el paralelo de $23^{\circ} 30^{\prime}$ y el de $26^{\circ}$, y los cochimíes tomaron la parte setentrional desde los $25^{\circ}$ hasta los 33 , y algunas islas del mar Pacífico. Cada una de estas tres naciones tenía su lengua propia.

A esta interpretación Francisco Javier Clavijero añadió los dialectos del idioma guaycura y señaló que "la guaicura tenia tantos dialectos diversos cuantas eran las ramas de la nacion que la hablaba, á saber: guaicuras propiamente dichos, aripas, uchitas, coras é indios de Conchó, llamados después lauretanos por el pueblo de Loreto que se fundó cerca de ellos" (ídem). Por su parte, Miguel del Barco (1988 [1757], 173-174) documentó la existencia de dos naciones que fueron la nación de pericúes y la nación de guaycuras, así como también lo hiciera con los subgrupos de la nación guaycura respecto a los que añadió que "estos guaycuros de La Paz son los que llaman callejúes, nombre particular de su ranchería, y no de nación distinta de los guaycuros". De otro lado, se tienen los textos del padre Miguel Venegas que coincidió con Francisco Javier Clavijero en afirmar que existen las tres lenguas (Venegas, 1757, 63).

Hay que agregar, además, acerca de este idioma, que la documentación hecha por el padre Juan Jacobo Baegert (1979 [1771], 9899) se constituye en otro ejemplo de los escasos testimonios y testigos de las lenguas indígenas, en su caso, la lengua guaycura. El padre Juan Jacobo Baegert es autor de las Noticias de la península americana de California, texto en el que escribió que este idioma carecía de preposiciones, pronombres, conjunciones, adverbios, imperativos, comparativos y superlativos, artículos o metáforas; razón por la cual su libro ha llegado a ser conocido como el "libro negro de la Baja California" por contener información falsa, considerar bárbaras tanto la lengua como la nación guaycura, y dar a entender que la lengua carece de palabras abstractas y que la nación no tiene la capacidad de progresar cultural e intelectualmente (Leedom Shaul, 2020, 1). 
También en el ámbito académico encontramos muy pocos estudios sobre estas lenguas, aunque destaca un texto hecho por el autor Leedom Shaul (2020). Motivado por hacer una recopilación más completa de los datos referidos a las lenguas indígenas que se hablaban en la Baja California, estudió las tres lenguas: pericú, cochimí y guaycura.

En su texto, en primer lugar, el autor Leedom Shaul (2020, 6-21, 70-96) se interesó por estudiar la lengua cochimí que resulta ser la más conocida en la actualidad porque al alcance de los investigadores se hallan los datos referentes a esta lengua desde la fonética hasta el léxico. Se tiene conocimiento de que la lengua cochimí tenía: un sistema de cinco vocales; vocales dobles; orden secuencial de morfemas $\mathrm{V}, \mathrm{CV}$ o CVC; palabras compuestas; casos de la sustantivación; sufijos; posesivos; formación de comparativos y superlativos; orden de la oración simple (sujeto-objeto-verbo); y caso ergativo-absolutivo. Cabe destacar que, en el plano léxico, el vocablo medesá, o 'paloverde' que se utiliza en el corpus de Isidro de Atondo y Antillón es de esta lengua (Leedom Shaul, 2020, 93).

Con posterioridad, el autor Leedom Shaul estudió la lengua guaycura, sobre la que apenas se tienen datos (Leedom Shaul, $2020,32)$. Entre la información que este autor destaca se halla su sistema de cuatro vocales (todas excepto la vocal [o]), su caso ergativo-absolutivo y el posible orden de morfemas igual que el de la lengua cochimí (V, CV, CVC).

Por último, Leedom Shaul estudió la lengua pericú (Leedom Shaul, 2020, 37-38; León-Portilla, 2000, 101). De esta lengua solo se conoce su sistema de cinco vocales, su articulación posiblemente aspirada y los vocablos "[...] ipiri-ka (axe), ka (big), itaurigui (chief, captain), nacui (conch), unoa (give), ауnu (fish), guaxoro (friend), uriuri (go, come), ipiri (knife), boox/boxo (pearl), utere (sit), miñicari (sky, heaven), eni (water)" ["ipiri-ka (hacha), ka (grande), itaurigui (capitán), nacui (concha), unoa (dar), aynu (pescado), guaxoro (amigo), uriuri (andar), ipiri (cuchillo), boox/ boxo (perla), utere (sentarse), miñicari (cielo), eni (agua)"] (Lee- 
dom Shaul, 2020, 38). La mayoría de las palabras documentadas por Leedom Shaul son recogidas también por León Portilla (1976, 93-95).

La lengua pericú es la más desconocida. Los primeros testimonios que existen sobre la misma son muy escasos ${ }^{7}$. Ya se han comentado en las líneas precedentes los de los frailes jesuitas (León-Portilla, 1976, 92-93). Veamos ahora los textos conocidos de los navegantes del siglo XVII. A este grupo pertenecen el testimonio de Pedro Porter y Casanate fechado a 24 de enero de 1649, cuyo vocabulario se desconoce; el escrito titulado Relación que dio el piloto Esteban Carbonel al virrey de Nueva España, marqués de Cerralvo, en México, a 30 de septiembre de 1632, testimonio que recoge vocablos de la lengua "ipiri: cuchillo, ipirica: hacha, uriuri: andar, utere: sentarse, unoa: dar aquello, boox: perla, nacui: concha, itaurigui: capitán" (Leedom Shaul, 2020, 93); y una carta del capitán Diego de la Parra fechada en el año 1683 y dirigida a Isidro de Atondo y Antillón, la cual da testimonio del uso de los vocablos "eni: agua, boxo: perla, aynu: pescado" (Leedom Shaul, 2020, 93-94).

\section{Edición crítica}

Los ejemplos contextualizados sobre el uso de estas voces se presentan en una edición propia que sigue las normas de la Red Internacional CHARTA, para cuya comprensión se recomienda vid. sus criterios (CHARTA, 2013, 7-15, 20-39); no obstante, para brindarles mayor comodidad a los lectores de este escrito vamos a repasarlos. Antes de proceder a su exposición primero hay que aclarar que en este escrito no se procede a la realización de la transcripción paleográfica por ser suficiente la edición crítica para el análisis léxico.

7 "Sources of information and contemporary research about the extinct Indian languages of Lower California are considerably limited. Above all this is true in the case of the Pericu language spoken by the natives who inhabited the southernmost portion of the peninsula" (León-Portilla, 1976, 100-101). 
De conformidad con estos criterios, las hojas y las líneas se han colocado entre llaves, por ejemplo, $\{\mathrm{h} 5 \mathrm{v}\},\{18\}$ y las abreviaturas son desarrolladas $(d i d<i>u)$. Las hojas corresponden al texto transcrito con independencia de la numeración del original. Las palabras difíciles de reconocer se han transcrito con tres puntos entre paréntesis puntiagudos, como sucede en el caso de la única palabra de este tipo que se halló en el corpus, concretamente en la línea 18 del folio 862v del Descubrimiento en Las Californias. La edición se ha realizado según las normas de acentuación actuales y se han simplificado las letras dobles. Adicionalmente, se ha utilizado el signo (') donde hay pérdida de una vocal ( $a^{\prime}$ ste paraxe); se transcriben las letras omitidas entre paréntesis puntiagudos, en cursiva (pesca< $n>$ do); y se editan las grafías $b, v, s, c, c ̧,-s s^{-}, g$, $j, x$ y otras de acuerdo con las normas: passava, assí, bajavan, berde, bauptiçado, mugeres, magestad, gentiles, balle, etc.

\section{Vocablo: aynu}

De acuerdo con lo comentado en las líneas anteriores, aynu es un vocablo pericú poco documentado con el cual se identificaba el pescado y fue registrado por Diego de Parra en el año 1683 como uno de los vocablos que empleaban los indígenas que habitaban las islas Espíritu Santo y San José (Rodríguez Tomp, 2002, 172; León-Portilla, 1976, 93-94; Ibarra Rivera, 2011, 63; Leedom Shaul, 2020, 38). Ello quiere decir que estas documentaciones hechas por los autores cuyos nombres se encuentran en el paréntesis, así como el testimonio de Diego de Parra, junto con los folios de Isidro de Atondo y Antillón que son transcritos seguidamente, son los únicos registros del vocablo aynu que se han encontrado hasta la fecha, al cual se suma el vocablo boxo/boox (vid. el epígrafe 6.1); salvo que boox también ha sido documentado en el escrito Relación que dio el piloto Esteban Carbonel al virrey de Nueva España, marqués de Cerralvo, en México, a 30 de septiembre de 1632 de Esteban Carbonel, como ya se ha indicado.

En los textos de Isidro de Atondo y Antillón el vocablo solo aparece una vez, concretamente en las Cartas del virrey marqués de 
la Laguna, donde se comprueban tanto el uso de la definición del vocablo, en el folio 34 (líneas 9 y 10), como su procedencia de la lengua de los indios de la isla del Espíritu Santo (pericú), en los folios 33 (línea 10) al 34. Este es el caso de la siguiente ocurrencia:

Y habiendo buelto 8 , dijeron $\{8\}$ habían andado registrando $\{9\}$ todas las ensenadas asta la punta $\{10\}$ de la isla; y que no hallaron $\{11\}$ comedero ninguno; que una de las $\{12\}$ dichas ensenadas tenía un puerto $\{13\}$ abrigado de todos vientos; y que $\{14\}$ passava a desembocar a la otra $\{15\}$ vanda de la mar, pero que $\{16\}$ no podía entrar desde el $\{17\}$ puerto adelante navío ninguno $\{18\}$ por haber poca agua; que, $\{19\}$ habiendo saltado en la $\{20\}$ playa, se les incorporaron $\{h 21\}\{1\}$ doce indios ${ }^{10}$ de dicha ranchería, $\{2\}$ que estaban pesca $<n>-$ do y que les $\{3\}$ enseñaron los cuchillos, ma\{4\}chetes y achas y les dijeron por $\{5\}$ señas que si les enseñaban donde $\{6\}$ había conchas se los darían, a que $\{7\}$ respondieron con palabras y $\{8\}$ señas que por allí no las había, $\{9\}$ que ellos venían a buscar $a y n u,\{10\}$ que assí llaman al pescado; $\{11\}$ y que en dicha playa vieron $\{12\}$ algunos ranchos desamparados $\{13\}$ y junto de ellos un pozo de agua $\{14\}$ salobre muy berde. (Cartas del virrey marqués de la Laguna, ff. 33-34)

\subsection{Boxo}

$\mathrm{Al}$ igual que el vocablo aynu, boxo 'perla' es un vocablo poco documentado de la lengua pericú. Este vocablo fue registrado antiguamente como boox por Esteban Carbonel en el año 1632 y como boxo por Diego de Parra en el año 1683 (Rodríguez Tomp, 2002, 172; León-Portilla, 1976, 93-94; Leedom Shaul, 2020, 38); en años más recientes la palabra ha sido documentada por Ibarra Rivera $(2011,65)$ con variantes boxoo, boxo, boo y boox, de las cuales la primera es la que se utiliza en este corpus.

8 Los soldados de don Isidro de Atondo y Antillón.

9 La isla del Espíritu Santo.

10 Los edúes (pericúes). La palabra pericúes no se aplica a lo largo del corpus y edú solo se aplica con referencia a la nación guaycura, es decir, en estos folios se usa el sustantivo indio para aludir a quienes son pericúes. 
El vocablo boxo es señalado por las bases de datos CORDE y CORDIAM (s. v. boxo), sin relación con la lengua pericú, sino que actúa como un marinerismo (DLE, s. v. bojo, marinerismo, quizás procedente del catalán, que designa 'bojeo' o 'perímetro o circuito de una isla o cabo'), en un documento del 1619 según el CORDE, y en la Historia verdadera de Bernal Díaz del Castillo (c 1568,28 ) según el CORDIAM ("y esto yo lo vi y anduve quando bolví terçera vez con Cortés; y terná de boxo esta isla obra de dos leguas").

La presencia del vocablo boxoo en este corpus es minoritaria, con un caso de su uso, esto es, en los folios 35 al 36 de las Cartas del virrey marqués de la Laguna, donde constatan no solo la definición de esta palabra proporcionada en el primer párrafo de este epígrafe, sino también su procedencia de la lengua de los indígenas de la isla de San José, pericú (vid. el epígrafe 6):

$\{18\}$ En seis ${ }^{11}$ nos hicimos a la vela en $\{19\}$ demanda de la isla de San Josef, $\{20\}$ donde dimos fondo detrás de $\{21\}$ la punta en paraje que haze $\{$ h 23$\}\{1\}$ puerto cerrado con la tierra $\{2\}$ firme. Y, como a las dos de la $\{3\}$ tarde, vinieron veinte indios ${ }^{12}\{4\}$ y comensaron a gritar y hazer $\{5\}$ señas fuésemos a tierra, que $\{6\}$ traían boxoo, o que assí llaman $\{7\}$ a las perlas, pidiendo cuchillos.

\subsection{Riyero}

La obra de Ibarra Rivera $(2011,106)$ es el único registro de la palabra riyero 'perla', que se ha encontrado hasta el momento, junto con los folios de don Isidro de Atondo y Antillón. Riyero fue documentado como un vocablo que identificaron indios (guaycuras) de San Ignacio de Loyola, llamado Digurupu en la lengua de los guaycuras, tal y como lo comprueba el último fragmento aquí transcrito (vid. el epígrafe 6.5. Guaycura. Monqui).

En este corpus se documenta el mismo número de ocurrencias del vocablo riyero que el que se halló de los vocablos peri-

11 En agosto de 1685.

12 Los edúes (pericúes). Vid. la nota 10. 
cúes aynu y boxoo, y, en las líneas 4 hasta la 11 del folio 56 de las Cartas del virrey marqués de la Laguna, se puede ver el significado de la palabra proporcionado en los párrafos precedentes, así como su procedencia de la lengua guaycura, dado que la ranchería San Matías está en los alrededores de Digurupu (tal y como lo comprueba el último fragmento aquí transcrito, para lo cual se recomienda vid. el epígrafe 6.5):

$\{21\}$ En veinte y tres fui yo, dicho $\{22\}$ almirante, con los soldados \{h 43\} \{1\} Simón de Sandoval y Francisco \{2\} de Aguilar, con dicho contramaestre, $\{3\}$ bussos y grumetes a reconoser la $\{4\}$ costa y ensenada de la ranche\{5\}ría de San Matías. Y habién\{6\}doles mostrado a los indios ${ }^{13}$ y $\{7\}$ mugeres de dicha ranchería achas, $\{8\}$ machetes y cuchillos, haziéndoles $\{9\}$ señas se los daríamos por perlas, $\{10\}$ que en la idioma de estos se lla $\{11\}$ man riyero, nos respondieron que $\{12\}$ no las tenían y señalaron que $\{13\}$ en una isla que teníamos $\{14\}$ enfrente, que distaría de la tierra $\{15\}$ firme como media legua, las había. (Cartas del virrey marqués de la Laguna, ff. 55-56)

\subsection{Medesá}

Frente a los vocablos anteriores, contrasta el uso del vocablo medese que aparece en un buen número de casos en el Descubrimiento en Las Californias. Los Vocablos indígenas de Baja California Sur (Ibarra Rivera, 2011, 80, 95) registran las formas medese y medesá, nombre cochimí que en la lengua de los monquis sería equivalente a dipuá, denominación con la que se identificaban los paloverdes, árboles leguminosos de la península de los que se obtienen las semillas como alimento. El autor Francisco Javier Clavijero (1980 [1852], 7) documentó el uso de medesá y dipuá, y en el siguiente fragmento proporciona una descripción detallada:

El medesá es un árbol grande que no da fruto todos los años, y en los lugares altos casi nunca: su tronco tiene la corteza verde

13 Los edúes (guaycuras). 
blanquizca, sus hojas son pocas y delgadas, y su fruto semejante al frijol, encerrado en pequeñas vainillas. Este fruto es muy apreciado por los indios, los cuales le mondan y le tuestan para comerle después en el invierno. Los bueyes comen bien las ramas tiernas de este árbol, pero su madera no sirve más que para leña. En Loreto le dan los indios el nombre de dipuá.

El corpus de los textos elaborados por Isidro de Atondo y Antillón señala la palabra (variante: medese) en los fragmentos de abajo (donde se corrobora su procedencia de la lengua cochimí), en los cuales se hallan otras voces propias de América (pitahayas). En ellos se muestra también una buena imagen de la religión de dichos indios cochimíes, la cual coincide con la descripción de Francisco Javier Clavijero (1980 [1852], 29-30) ${ }^{14}$. A continuación, se presentan los fragmentos donde se pueden observar los casos de los contextos mencionados:

Los días pasados descubrimos que $\{16\}$ idolatravan estos bár$\operatorname{varos}^{15}$, porque en el Real de $\{17\}$ San Isidro, que dista de este ${ }^{16}$ tres leguas $[\ldots]$, se juntaron cerca $\{20\}$ de tres mil almas y tubieron grandes super\{21\}tiziones y hicieron grande benerazión, fiesta $\{\mathrm{h}$ $7 \mathrm{v}\}\{1\}$ y bailes a un ídolo del tamaño de un indio $\{2\}$ rezién nazido envijado a su usanza. A este lo \{3\} llevaron en procesión hombres y mugeres a un tol\{4\}do que le tenían hecho, adonde le corrieron gran $\{5\}$ des carreras y se dieron famosas artasgas de la $\{6\}$ semilla que llaman medese. Y este dizen dichos xen\{7\}tiles que les habla en

14 "Los cochimíes decian que en el cielo habitaba un gran señor [...], el cual crió el cielo, la tierra, las plantas, los animales, el hombre y la mujer. [...] Los cochimíes [...] hacian mencion de un hombre que en el tiempo antiguo vino del cielo á beneficiar á los hombres, y por esto le llamaban Tamá ambei ucambi tevivichi, esto es, el hombre venido del cielo [...]". El fragmento termina diciendo que la comunidad hace las fiestas con bailes, gritos, carreras y comida; y que sus miembros pintan al ídolo con varios colores.

15 El pueblo de cochimíes.

16 Real de San Bruno. 
su lengua que baxa $\{8\}$ del cielo que les da las pitahayas ${ }^{17}$, el medese $\{9\}$ y otras semillas que comen silvestres y que $\{10\}$ tiene un pie y dos dientes, uno abajo y otro $\{11\}$ arriba. (Descubrimiento en Las Californias, ff. 846r-846v)

Y que dicho bulto $\{20\}$ lo pusieron devaxo de dicha ramada, alto un $\{21\}$ poco del suelo y al pie un gran montón $\{22\}$ de la semilla que llaman medese [...]. Salían $\{3\}$ unos tras otros entreverados hombres y mugeres $\{4\}$ y davan una gran carrera; y el capitán en lle\{5\} gando al fin de ella con toda su gente parava $\{6\}$ junto a dicho bulto y empeçavan a hablar $\{7\}$ todos y a un mismo tiempo se bajavan haziéndole $\{8\}$ una umillazión y después descansavan como $\{9\}$ un cuarto de ora y bolvían a proseguir la $\{10\}$ misma carrera con las mesmas ceremonias [...]. Y, den\{16\}tro de breve rato, empezaron a cantar $\{17\}$ y continuaron todo el día con gritos y bailes $\{18\}$ a pausas. Y que, al ponerse el sol, se sentaron $\{19\}$ en rueda en barias partes y les co $\{20\}$ menzaron a repartir de dicha semilla me\{21\}dese, que tenían amontonada delante de dicho $\{22\}$ bulto. (Descubrimiento en Las Californias, ff. 888r-888v)

Y que, preguntándoles qué figura $\{13\}$ era aquella que tanto habían zelebrado, $\{14\}$ dieron a entender dichos gentiles que era el $\{15\}$ que les dava el mantenimiento y el que, cuan\{16\}do llueve, baxa del cielo a regalarlos y traerles $\{17\}$ las pitahayas y el medese y que ya se había $\{18\}$ buelto al cielo. (Descubrimiento en Las Californias, f. 890r)

\subsection{Didiu. Edú}

Las formas didios y didiu(s) se muestran en el documento Vocablos indígenas de Baja California Sur (Ibarra Rivera, 2011, 80). La primera forma, didios, denominaba respectivamente a los cochimíes que vivían en los territorios ubicados frente a la isla de San

17 DAMER y DEM (s. v. pitahaya): 'fruto de la pitahaya, de colores amarillo, rojo o morado, de forma redonda, con pulpa comestible de sabor agridulce, con espinas en la cáscara y, dentro, con semillas negras'. La pitahaya (planta, Hylocereus) que da este fruto es de las cactáceas, con hojas carnosas y verdes, tallo con espinas, flores blancas o de otros colores y pulpa comestible (DAMER, s. v. pitahaya; DLE, s. v. pitahaya; DEM, s. v. pitahaya). 
Ildefonso, es decir, en la provincia de San Andrés. La segunda, didiu, en singular, hacía referencia a un idioma califórnico, y en plural a todos los habitantes de dicha provincia. En lo que respecta a la palabra edú, el texto de Vocablos indígenas de Baja California Sur (p. 81) y el elaborado por Holmos Montaño (2018, 25-26) coinciden en que era utilizado por monquis para identificar a miembros de la comunidad de pericúes, incluso señalan que la palabra era empleada por cochimíes para referirse a miembros de la comunidad de guaycuras que vivían en el Puerto de Danzantes y otros sitios ${ }^{18}$ cerca de los de los didios.

Ambas palabras se recogen en el CORDE con su única aparición en la Historia de la Iglesia en la América española desde el descubrimiento hasta el siglo XIX, texto elaborado por el historiador y jesuita León Lopetegui (1965, 628-629), precisamente citado como fuente información recogida en este corpus:

Célebre la expedición en que Kino y Atondo (diciembre de 1684), atravesando por primera vez la península a lo largo del río de la Purísima, que llamaron de Santo Tomás, llegan hasta la costa del Pacífico. /// Los catorce meses que permanecen en California los emplean los españoles en explorar la tierra, civilizar a los nativos, aprender sus lenguas y cristianizarlos; aunque previendo lo precario de su establecimiento, no bautizaron sino a unos trece enfermos y moribundos. Los nativos se muestran más sociables y sumisos que los guaicuros del sur. Atraídos por la comida, a todas horas están dispuestos para la doctrina, y los niños no se apartan de la choza del padre. /// Con arduo trabajo aprenden los dialectos, que eran dos, a la puerta del real: al sur el edú llamado noe, a que se dedicó el padre Goñi, y al norte el didiu, llamando neve o cochimí, a que se aplicó el padre Kino.

18 El mapa Delineación de la Nueva Provincia de S[an] Andrés, del Puerto de La Paz, y de las islas circumvecinas de Las Californias, ó Carolinas, del año 1683 y custodiado en el Archivo de Indias, y el folio 863r (líneas 11 y 12) del Descubrimiento (ver más abajo), corrobora que estos sitios eran territorios de los edúes (guaycuras). 
En el corpus que se tomó para elaborar este artículo se registran nueve apariciones de la palabra didiu (cuatro de ellas en el Descubrimiento en Las Californias, dos en los Autos sobre la última entrada en Las Californias y tres en las Cartas del virrey marqués de la Laguna); también se registran diez apariciones del vocablo edú (dos de ellas en el Descubrimiento, cinco en los Autos y tres en las Cartas). Allí se comprueban algunos datos como, por ejemplo, en el folio 863r del Descubrimiento se corrobora que las lenguas cochimí y guaycura son diferentes, y en el folio 982v de los $A u$ tos se comprueba que es posible que existan diferencias entre las lenguas guaycura y monqui, y el idioma, según Ibarra Rivera (2011, 97), desconocido:

Cer $\{13\}$ tifico y doy fee como el día veinte y seis de $\{14\}$ octubre de este presente año de seiscientos y ochenta $\{15\}$ y cuatro se sacaron de cinco fardos de la \{16\} limosna que su magestad mandó dar para los gentiles $\{17\}$ de este reino de la California cantidad de $\{18\}$ frezadas, güipiles ${ }^{19}$, enaguas ${ }^{20}$, cotones, tilmas ${ }^{21}\{19\}$ y sombreros, lo cual se les repartió a los $\{20\}$ hombres, mugeres y niños de las circum $\{21\}$ bezinas rancherías de nazión didiu, que $\{22\}$ avitan en este valle ${ }^{22}[\ldots]$. Asimismo certifico y doy fe como el día de $\{14\}$ San Carlos, cuatro de noviembre de este pre $\{15\}$ sente año de seiscientos

19 DLE (s. v. huipil; también güipil; náhuatl huipilli): “El Salv., Guat., Hond. y Méx. Especie de blusa adornada propia de los trajes indígenas", es decir, "Mx, Gu, Ho, $E S, N i$. Blusón largo, sin mangas y con adornos que visten las mujeres indígenas" (DAMER, s. v. huipil; también güipil o hipil; náhuatl huipilli). Más detalladamente puede significar "prenda con que las mujeres, en particular las de pueblos indios de México, se cubren el tórax; es generalmente de algodón o manta blanca, amplia, con o sin bordados, escotada y llega hasta la cintura o los muslos" (DEM, s. v. huipil).

20 Enaguas, en plural, de uso en México, 'prenda exterior femenina que cuelga desde la cintura' (DAMER, s. v. enaguas; DLE, s. v. enagua; DEM, s. v. enagua).

21 Sin registro en el DAMER, aunque sí es documentado por el DLE (s. v. tilma; náhuatl tilmatli) como sigue: "Méx. Manta de algodón que llevaban los hombres del campo a modo de capa, anudada sobre un hombro", o más detalladamente por el DEM (s. v. tilma) como "manta rectangular o cuadrada, larga y delgada, hecha de algodón, lana o ixtle, que se amarra por dos puntas sobre el hombro y que algunos campesinos utilizan a modo de abrigo".

22 Provincia de San Andrés. 
y ochenta y cuatro, $\{16\}$ habiendo hecho Salva Real y dicho la $\{17\}$ misa en nombre de nuestro católico $\{18\}$ rey, que Dios guarde, con la $<\ldots>$ de la $\{19\}$ limosna antezedente que se dio en $\{20\}$ nombre de su magestad a las rancherías $\{\mathrm{h} 2 \mathrm{r}\}\{1\}$ de la nazión didiu que havitan en $\{2\}$ este balle. Concurrió a este valle mucho $\{3\}$ gentilismo de la nazión $e d u ́$, que son de di\{4\}ferente lengua que los que havitan este $\{5\}$ balle; [...] $\{11\}$ los cuales ${ }^{23}$ havitan en el puerto de Dançan $\{12\}$ tes [...]. Y los días que suelen acudir $\{16\}$ a este real ${ }^{24}$, dichos padres ministros les en $\{17\}$ señan la dotrina cristiana y dos de dichos $\{18\}$ padres y un soldado travaxan en apren\{19\}der la lengua de dicha nazión edú y el \{20\} padre retor y el alférez Nicolás de Con\{21\}treras Ladrón de Guevara en aprender $\{\mathrm{h} 2 \mathrm{v}\}\{1\}$ la lengua de dicha nazión didiu, que es de $\{2\}$ la que tenemos noticia que se estiende hasta la \{3\} contracosta. (Descubrimiento en Las Californias, 862r-863v)

Que el día lunes seis $\{17\}$ de noviembre de este presente año de seiscientos y ochenta y $\{18\}$ cuatro, serían las doce del día, poco más o menos, $\{19\}$ estando en guarda de los cavallos que andavan $\{20\}$ pastando, vieron como el indio capitán de la $\{21\}$ nazión $\operatorname{did}<i>u$, a quien llamamos Leopoldo (aunque \{22\} no está bautiçado), subió a lo alto de un ze\{23\}rro bestido de una red de ylo toda poblada $\{\mathrm{h} 2 \mathrm{v}\}$ \{1\} de madejitas de cavellos [...]. (Descubrimiento en Las Californias, ff. 887r-887v)

Zertifico y doy fee como $\{4\}$ el día viernes diez y seis de $\{5\}$ febrero de este presente año $\{6\}$ de mil y seiscientos y ochen $\{7\}$ ta y cinco salimos del Real $\{8\}$ de San Bruno para ir $\{9\}$ la tierra adentro por diferen $\{10\}$ te parte y rumbo que la $\{11\}$ entrada antecedente que $\{12\}$ emprendí el año pasado $\{13\}$ de seiscientos y ochenta y $\{14\}$ cuatro yo, dicho almirante, [...] y algunos gentiles de la nazi $\{14\}$ ón edú que llevábamos por guía. (Autos sobre la última entrada en Las Californias, ff. 961v, 962v) 
$[\ldots]\{5\}$ al cual dicho puesto llaman $\{6\}$ los naturales ${ }^{25}$ Damadamuyete $^{26} .\{7\}$ Aquí armamos nuestro real y le $\{8\}$ pusimos por nombre la Con $\{9\}$ cepción por haber llegado en $\{10\}$ sávado. Y viendo que a dicho $\{11\}$ paraxe le cercavan serra\{12\}nías muy ásperas y altas, di orden $\{13\}$ a dicho ayudante fuese adelante $\{14\}$ a descubrir camino con los $\{15\}$ soldados Simón de Sandoval $\{16\}$ y Diego Días por la $\{\mathrm{h} 4 \mathrm{v}\}\{1\}$ por la inteligencia $\{2\}$ que este tiene de la len\{3\}gua de dicha nazión \{4\} edú. (Autos sobre la última entrada en Las Californias, ff. 964r-964v)

Es toda tierra baxa y $\{3\}$ dicha encenada ${ }^{27}$ está abrigada $\{4\}$ del norueste y norte, porque $\{5\}$ bienen por sobre la tierra y del $\{6\}$ norte está abrigada de la $\{7\}$ isla de Coronados, que distará como $\{8\}$ cuatro leguas y del leste y sueste $\{9\}$ con la isla del Carmen ${ }^{28}$. [...] A la noche llegó el \{4\} capitanejo de dicha nazión edú, aquí \{5\} en llamamos Dionisio, aunque no está $\{6\}$ bauptiçado y me regaló con un \{7\} poco de pescado. (Autos sobre la última entrada en Las Californias, ff. 971r-971v)

Llámanle los naturales a'ste paraxe $\{12\}$ Chuenqui $^{29}$. [...] Y dicha ranchería se $\{16\}$ compone de más de duzientas $\{17\}$ almas toda vien axestada y $\{\mathrm{h} 22 \mathrm{v}\}\{1\}$ dispuesta y algunas de mucha $\{2\}$ edad a quien con particularidad re\{3\}galamos. $Y$ dicho padre ministro $\{4\}$ reconoció eran dos parzialidades $\{5\}$ porque hablan distintas lenguas; la $\{6\}$ una es de la nación moquis [...] y la otra es de $\{10\}$ los edúes. (Autos sobre la última entrada en Las Californias, ff. 982r-982v)

Y habi\{2\}endo benido la noche antes $\{3\}$ el indio capitán de la $\{4\}$ nazión didiu, a quien llamamos Leopol\{5\}do (aunque no está baup-

25 Edúes (guaycuras).

26 Otro topónimo señalado por Ibarra Rivera (op. cit., 79) indica que al lugar se le dio el nombre de La Concepción por el almirante, tal y como se muestra en los folios citados.

27 San Dionisio.

28 Según lo indica el mapa Delineación de la Nueva Provincia de S[an] Andrés, del Puerto de La Paz, y de las islas circumvecinas de Las Californias, ó Carolinas, del año 1683, las islas de los Coronados y del Carmen se ubican frente al Puerto de los Danzantes, cabe recordar el territorio del pueblo de edúes (guaycuras).

29 Según Ibarra Rivera $(2011,79)$, era una ranchería del pueblo de monquis. 
tiçado), y $\{6\}$ preguntándole dónde venía, respondió que $\{7\}$ había andado en las tierras de sus $\{8\}$ parientes y todos presumimos que había $\{9\}$ ido a avisar a su gente de nuestra $\{10\}$ entrada. (Autos sobre la última entrada en Las Californias, f. 999v)

A lo $\{13\}$ primero asiento por punto prinzi $\{14\}$ pal que lo mexor de cuanto $\{15\}$ hemos visto se comprenden $\{16\}$ este valle y en el puesto de $\{17\}$ San Dionisio en donde se $\{\mathrm{h} 2 \mathrm{r}\}\{1\}$ podrán formar dos misiones de $\{2\}$ la nazión que llamamos edú, la \{3\} cual es muy estendida, y la otra $\{4\}$ en este dicho valle que avita $\{5\}$ la nazión didiu formando $\{6\}$ dos pueblos, el uno en el $\{7\}$ puesto de San Juan y el otro en $\{8\}$ el de San Isidro. (Autos sobre la última entrada en Las Californias, ff. 1066v-1067r)

Dimos fondo ${ }^{30}$ en $\{19\}$ dicha vaya ${ }^{31}$ como a las $\{20\}$ doce del día, adonde vinieron $\{21\}$ catorse indios en tres valzas [...]. Y habién $\{4\}$ doles buelto a enseñar perlas, $\{5\}$ achas, cuchillos y machetes $\{6\}$ dándoles a entender se los daría\{7\}mos por dichas perlas, asían de $\{8\}$ mostraziones con suspiros sintiendo $\{9\}$ no tenerlas para conseguir dichas $\{10\}$ erramientas y nos dieron a entender $\{11\}$ estos naturales tenían guerras con $\{12\}$ los de la nazión edú. (Cartas del virrey marqués de la Laguna, ff. 43-44)

Digo que el $\{15\}$ valle de San Bruno es avita $\{16\}$ do de dos distintas naziones, la $\{17\}$ una llaman didiu y esta avi\{18\}ta la mayor parte $\{$ h 2$\}\{1\}$ de dicho valle y se estien $\{2\}$ de la tierra adentro $\{3\}$ hasta la contracosta y mar $\{4\}$ de Filipinas y comprende $\{5\}$ en sí el valle que llama\{6\}mos de San Juan; la otra lla\{7\}mada edú, esta avita parte de $\{8\}$ dicho valle, se estiende vein $\{9\}$ te y cinco leguas, poco más $\{10\}$ o menos, por la costa y confina, $\{11\}$ según hemos tenido notizia $\{12\}$ de ellos mismos por dicha contra\{13\}costa, con la nazión guicura $\{14\}$ del puerto de La Paz ${ }^{32}$. (Cartas del virrey marqués de la Laguna, ff. 127-128)

30 Isidro de Atondo y Antillón con su gente.

$32 \mathrm{Vid}$. la nota 2 o el epígrafe 6.5, es decir, quienes son guaycuras de La Paz se llaman callejúes, voz no utilizada a lo largo del corpus del almirante. 
$\{9\}$ Los párbulos y adultos de la $\{10\}$ nazión didiu, que son los $\{11\}$ que dominan y señorean la $\{12\}$ parte donde está fabricado $\{13\}$ el Real de San Bru\{14\}no, eran los más asisten\{15\}tes a acudir a tarde y $\{16\}$ a mañana a la dotrina, $\{17\}$ que les enseñavan los padres $\{\mathrm{h}$ $5\}\{1\}$ misioneros a rezar el Rossa\{2\}rio y las letanías y cantan $\{3\}$ la Salve. (Cartas del virrey marqués de la Laguna, ff. 130-131)

Para la reduzión y con $\{6\}$ versión de los naturales de dicho $\{7\}$ valle de San Bruno y sus $\{8\}$ circunvezinas rancherías, son $\{9\}$ nezessarios cuatro padres mi\{10\}nistros, dos que eduquen y dotri\{11\}nen la nazión didiu y dos para $\{12\}$ la enseñanza de la nazión $\{13\}$ edú, porque, si falleze o enferma $\{14\}$ el uno, no se queden los $\{15\}$ naturales sin ministro $\{16\}$ y se pierda lo que se hubiere $\{17\}$ adquirido de su idioma. (Cartas del virrey marqués de la Laguna, f. 144)

\subsection{Guaycura. Monqui}

Bajo la anterior entrada, en la línea 13 del folio 128 de las Cartas del virrey marqués de la Laguna se registró el único caso donde aparece la palabra guaycura (forma: guicura) a lo largo del corpus. Este vocablo, con las formas guaycura, waicura, huaicura, waicuri y waikuri (Ibarra Rivera, 2011, 85-86, 114), se utiliza para designar a indios localizados entre las comunidades de cochimíes y pericúes, con los subgrupos coras, monquis, aripes, uchitíes, guaycuras, o, en La Paz, callejúes.

En el CORDE aparece la palabra guaycura en la ya mencionada Historia de la Iglesia en la América española desde el descubrimiento hasta el siglo XIX de autoría de León Lopetegui (1965, 227: “La estirpe guaycura-pericú ocupaba la mitad sur de Baja California"), así también se muestra la palabra guaicuros en la página 629 de esta obra (vid. 6.4. Didiu. Edú). Tampoco faltan ejemplos de la palabra en el DEM (s. v. guaycura, guaicúra) donde se define como "pueblo amerindio que habitó al sur de la península de Baja California, hoy desaparecido; se desconoce su lengua y la relación que haya tenido con otros pueblos de la región", y en su función de adjetivo o sustantivo también se define como "que pertenece a este pueblo o se relaciona con él". 
En lo que respecta a la palabra monqui, en los Vocablos indígenas de Baja California Sur de Ibarra Rivera $(2011,97)$ se pueden apreciar las formas mogis, moqui y monqui(s), con las cuales cabe recordar se identifica a los indígenas de Loreto. En el CORDIAM (s. v. moqui, 'provincia de Moqui') se indica que esta palabra se usó a lo largo de los siglos XVII y XVIII tanto en México como en los Estados Unidos.

Frente a guicura, la palabra moquis (de monquis) se documenta en más ocasiones en el corpus de Isidro de Atondo y Antillón, concretamente en los folios 975r, 982v (en la línea 6, vid. la anterior entrada) y $985 \mathrm{r}$ de los Autos sobre la última entrada en Las Californias. Son los casos de las siguientes ocurrencias:

Este día ${ }^{33}\{2\}$ andaríamos como tres leguas $\{3\}$ y armamos nuestro real en $\{4\}$ un arroyo cerca de la playa $\{5\}$ de una ranchería que los $\{6\}$ naturales llaman Nautré34. Pusimos\{7\} le por nombre San Pedro [...]. En esta ranchería $\{2\}$ hallamos más de trezientos in $\{3\}$ dios de arco y flechas y mucha $\{4\}$ turba de párbulos [...]. Y así estos $\{9\}$ gentiles ${ }^{35}$ como los que nos habían $\{10\}$ seguido de la ranchería de Santa $\{11\}$ Águeda nos dijeron que nos $\{12\}$ bolviésemos, que no prosiguiésemos $\{13\}$ adelante porque se seguía otra $\{14\}$ nazión que era muy numerosa, $\{15\}$ a los cuales llaman moquis y $\{16\}$ que tenían sus rancherías por don\{17\}de habíamos de ir. (ff. 974v-975r)

\{17\} Salimos de San Matías \{18\} y en nuestra compañía \{h 23r\} $\{1\}$ algunos naturales ${ }^{36}$ de dicha $\{2\}$ ranchería [...]. Proseguimos por una $\{6\}$ barranca de ceburrucal y por $\{7\}$ ella caminamos como media le\{8\}gua hasta llegar a un $\{9\}$ llano, donde salió a rezivirnos $\{10\}$ una ranchería de más de $\{11\}$ cincuenta indios; y a dicho $\{12\}$ puesto

33 El 22 de febrero del año 1685.

34 Otro de los topónimos documentados por Ibarra Rivera (2011, 99-100), Nautré o Notrí, hace referencia a una ranchería a la que los españoles le dieron el nombre de San Pedro, como se indica en estos folios.

35 Edúes (guaycuras).

36 Edúes (guaycuras). 
llaman los naturales $\{13\}$ Digurupu. [...] Pusimosle por nom $\{2\}$ bre San Ignazio de Loyola. [...] Andaríamos $\{13\}$ este día como dos leguas y ar\{14\}mamos nuestro real a la $\{15\}$ orilla de la mar en un $\{16\}$ arroyo seco que los naturales $\{17\}$ llaman Taraypua ${ }^{37}$. Pusimos $\{18\}$ le por nombre San $\mathrm{Ba}\{19\}$ lerio por haber llegado en $\{\mathrm{h} 24 \mathrm{v}\}\{1\}$ su día. [...] Y $\{12\}$ tubieron mucho pasto las bes $\{13\}$ tias en este paraje y se $\{14\}$ despidieron las guías ${ }^{38}$ de las $\{15\}$ rancherías antezedentes dizi\{16\}éndonos con señas y palabras $\{17\}$ no pasavan adelante, $\{18\}$ porque la nazión que se $\{19\}$ seguía es la que llaman moquis $\{\mathrm{h} 25 \mathrm{v}\}$ $\{1\}$ y son sus enemigos, y que el $\{2\}$ día siguiente al ponerse el $\{3\}$ sol yendo playa a playa, llegaría $\{4\}$ mos al primer paraje donde $\{5\}$ había agua y algunos\{6\} manchones de carrizo. (ff. 982v-985v)

\section{Conclusiones}

Los resultados hallados hacen evidente, en primer lugar, la limitada documentación que se halla en los testimonios tempranos y en las bases de datos tomadas en cuenta para este artículo sobre las lenguas desaparecidas pericú, cochimí y guaycura, pues, por ejemplo, las palabras mencionadas en este estudio solo son documentadas por el CORDE, el CORDIAM, el DEM o por Ibarra Rivera; así también, el estudio hace evidente, en segunda instancia, la escasa investigación académica que se ha desarrollado sobre estas lenguas, dado que solo se encontraron los trabajos realizados por León Portilla, Leedom Shaul, Holmos Montaño o Golla. De otra parte, se ha podido observar que entre todas las palabras estudiadas aynu, boxoo y riyero son, al igual que guaycura (variante gráfica: guicura), las que son menos documentadas en el corpus del almirante de Las Californias don Isidro de Atondo y Antillón; frente a las palabras didiu (9 resultados) y edú (10 resultados), o en menor medida frente a las palabras medese (5 resultados) y moquis (3 resultados).

37 Otro de los topónimos de Ibarra Rivera $(2011,108)$ del arroyo al que dieron el nombre de San Valerio.

38 Edúes (guaycuras). 
Otro resultado que resalta en el estudio es que los vocablos pericúes hallados en las Cartas del virrey marqués de la Laguna, como su único registro en todo el corpus del almirante, identifican el pescado (aynu) o las perlas (boxoo). El vocablo guaycura, riyero 'perla', también es de uso minoritario, dado que en dichas Cartas es donde se halla su único registro. En cuanto al vocablo cochimí, medese o medesá, se registró en el corpus en el Descubrimiento en Las Californias donde se usó con el significado de 'la semilla del paloverde'.

Asimismo, han sido objeto de estudio en este artículo las palabras con las cuales se designan las tribus bajacalifornianas edú, didiu, monqui y guaycuras, que fueron registradas en todo el corpus. En primer lugar, se encontró que los pueblos cochimíes y monquis utilizaban la palabra edú respectivamente para aludir a las comunidades de guaycuras y de pericúes, identificaciones de las cuales la primera se utilizó en el corpus. Con el segundo vocablo mencionado, didiu, se designaba a un subgrupo de la nación cochimí que se ubicaba cerca de los territorios de edúes (guaycuras). La palabra guaycura se usó en el corpus en relación con la nación que se ubica entre el pueblo de cochimíes ubicado en el norte y el pueblo de pericúes ubicado en el sur. Finalmente, se pudo advertir que la palabra monquis designa a miembros guaycuras de Loreto, conocidos como loretanos.

En resumen, en estas páginas se propuso examinar los vocablos de las lenguas extintas documentadas en los textos castellanos del siglo XVII y de autoría de don Isidro de Atondo y Antillón, almirante de Las Californias nacido en Navarra, estudio que se ha complementado con el de las palabras igualmente importantes con las cuales se designan las naciones observadas en sus textos, todas bajacalifornianas y de cuyas lenguas proceden los vocablos aynu, boxo, riyero o medesá. Resultado de este estudio aquí documentado es poner de manifiesto la escasa documentación existente hasta la actualidad sobre dichas lenguas y palabras, tanto en los testimonios de navegantes o misioneros de la época como en los estudios académicos que se han realiza- 
do hasta el momento. Con todo lo anterior se ha cumplido otro objetivo secundario de este análisis, consistente en clarificar los posibles sentidos de habla que las voces tuvieran a la luz de los contextos y ofrecer ejemplos amplios del uso de estas voces con las fuentes del corpus tomado para este estudio.

\section{Referencias bibliográficas}

Academia Mexicana de la Lengua (s.f.) Corpus Diacrónico y Diatópico del Español de América (CORDIAM). (www.cordiam. org).

Asociación de Academias de la Lengua Española (2010) Diccionario de Americanismos (DAMER), Madrid, Santillana. (http://lema.rae.es/damer/).

Consejo de Indias. (1685) Autos sobre la última entrada en Las Californias, Sevilla, Archivo General de Indias (Patronato, 31, R. 8). (http://www.mecd.gob.es).

BAEgert, JoHAnn JaCoB. (1979) Observations in Lower California (translated from the original German [Nachrichten von der Amerikanischen Halbinsel Californien, 1771]), Berkeley, University of California Press. (En línea, https://publishing.cdlib.org/ucpressebooks/view?docId=ft5r29n9xv).

Busto-Ibarra, Karina (2015) "Historical Archaeology of Baja California Sur", Pacific Coast Archaeological Society Quarterly, 51 (3/4), 91-116.

CartasdelvirreymarquésdelaLaguna(1686)Sevilla,Archivo General de Indias (México, 56, R. 1, N. 1). (http://www.mecd. gob.es).

Clavijero, Francisco Javier (1980) Historia de la Antigua o Baja California. Obra póstuma del padre Francisco Javier Clavijero, de la Compañía de Jesús (reproducción digital del original de 1852), Cambridge, Mass., Harvard College Library Digital Imaging Services. https://digitalcollections.library.harvard.edu/catalog/990044169030203941.

Consejo de Indias (1863) Delineación de la Nueva Provincia de S[an] Andrés, del Puerto de La Paz, y de las islas circumvecinas de 
Las Californias, ó Carolinas [...], Sevilla, Archivo General de Indias (MP-MEXICO, 76). (http://www.mecd.gob.es).

Del Barco, Miguel (1988) Historia natural y crónica de la Antigua California [adiciones y correcciones a la noticia de Miguel Venegas] (edición y estudio preliminar de Miguel León-Portilla; reproducción del original de 1757), México, UNAM, Instituto de Investigaciones Históricas.

Descubrimiento en las Californias (1685) Sevilla, Archivo General de Indias (Patronato, 31, R. 7). (http://www.mecd.gob.es).

El Colegio de México Diccionario del español de México (DEM), México, El Colegio de México, A.C. (https://dem.colmex.mx/).

Golla, Víctor (2011) California Indian languages, Berkeley, University of California Press.

González, Pedro (1994) “El Archivo General de Indias y su proyecto de informatización. Nuevas posibilidades de investigación", Cuadernos de Historia Moderna, 15, 231-250.

Hernández Aparicio, Pilar (1980) "Los viajes de don Isidro de Atondo y Antillón a California 1683-86", Anuario de Estudios Americanos, (37), 3-43.

Holmos Montaño, Francisco (2018) Apuntes cronológicos de Baja California Sur y Los Cabos. Recopilación, La Paz, México, Instituto Sudcaliforniano de Cultura.

Ibarra Rivera, Gilberto (2011) Vocablos indígenas de Baja California Sur, La Paz, México, Gobierno del Estado de Baja California Sur. http://archivohistorico.bcs.gob.mx/vocablos/vocablos.pdf

Laylander, Don (1985) "Some Linguistic Approaches to Southern California's Prehistory", San Diego State University Cultural Resource Management Center Casual Papers, 1, 14-58. (En línea, https://www.academia.edu).

Lázaro de la Escosura, Pilar y Ceballos Aragón, Isabel María (2008) Archivo General de Indias, Madrid, Ministerio de Cultura.

Leedom Shaul, David (2020) Baja California Languages: Description and Linguistic Prehistory, Berkeley, University of California. (https://escholarship.org/uc/item/3w42j7x8). 
León-Portilla, Miguel (1976) "Sobre la lengua pericú de la Baja California", Anales de Antropología, 13(1), 87-101.

León-Portilla, Miguel (2000) La California mexicana. Ensayos acerca de su historia, México, UNAM, Instituto de Investigaciones Históricas.

Mathes, W. Michael (1969) “A Biographical Note on Isidro de Atondo y Antillón, Admiral of theCalifornias", California Historical Society Quarterly, 48(3), 211-218.

(https://www.semanticscholar.org).

Real Academia Española Corpus Diacrónico del Español (CORDE). (http://corpus.rae.es/cordenet.html).

Real Academia Española (2014) Diccionario de la Lengua Es-

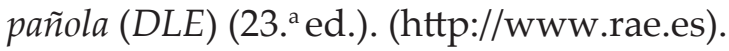

Red Internacional CHARTA (2013) Criterios deedición de documentoshispánicos(OrígenessigloXIX) delaRedInternacional CHARTA. http://files.redcharta1.webnode.es/200000023de670df5d6/Criterios\%20CHARTA\%2011abr2013.pdf.

Rodríguez Tomp, Rosa Elba (2002) Cautivos de Dios. Los cazadores-recolectores de Baja California durante el periodo colonial, Tlalpan, Instituto Nacional Indigenista.

Rodríguez Tomp, Rosa Elba y Almada, Rossana (2014) “Protagonistas de la exploración y colonización de la península de California. Una revisión de la colección "Californiana"', Meyibó: Revista de Investigaciones Históricas, 8, 75-102.

Rojas García, Reyes (coord.) (2016) Archivo General de Indias: El valor del documento y la escritura en el Gobierno de América, Madrid, Ministerio de Educación, Cultura y Deporte. (https://www. libreria.culturaydeporte.gob.es).

Survey of California and Other Indian Languages (2019) Map search, Berkeley, University of California. (https://cla.berkeley.edu/cla-map.php).

Venegas, Miguel (1757) Noticia de la California y de su conquista temporal, y espiritual hasta el tiempo presente. Sacada de la Historia manuscrita, formada en México año de 1739. por el padre Miguel Venegas, de la Compañia de Jesús; y de otras noticias y relaciones antiguas, 
y modernas. Añadida de algunos mapas particulares, y uno general de la America Septentrional, Assia Oriental, y Mar del Sur (...), Madrid, Imprenta de la Viuda de Manuel Fernández y del Supremo Consejo de Inquisición. (https://books.google.es/).

Wilken, Michael alan (2009) “Baja Languages Face an Uncertain Future", News from Native California, 22 (3), 5-7, 30-31. 
